\title{
Heteroclinic Switching in Coupled Oscillator Networks: Dynamics on Odd Graphs
}

\author{
Peter Ashwin, Gábor Orosz, and Jon Borresen
}

\begin{abstract}
We review some examples of dynamics displaying sequential switching for systems of coupled phase oscillators. As an illustration we discuss a simple family of coupled phase oscillators for which one can find robust heteroclinic networks between unstable cluster states. For $N=2 k+1$ oscillators we show that there can be open regions in parameter space where the heteroclinic networks have the structure of an odd graph of order $k$; a class of graphs known from permutation theory. These networks lead to slow sequential switching between cluster states that is driven by noise and/or imperfections in the system. The dynamics observed is of relevance to modelling the emergent complex dynamical behaviour of coupled oscillator systems, e.g. for coupled chemical oscillators and neural networks.
\end{abstract}

\section{Introduction}

Coupled phase oscillator networks provide a set of models that are very useful in a variety of applications ranging from theoretical and computational neuroscience $[10,11,17,18]$ to coupled chemical reactors [21]. They provide models that are amenable to variety of approaches aimed at understanding the emergent phenomena of such nonlinear dynamical systems. These approaches include "continuum approximations" as well as detailed studies of the dynamics and bifurcations of small numbers of oscillators; see [31] for a review. This paper reviews some recent results on switching dynamics for small numbers of oscillators. It is also explained how one of the dynamical structures (heteroclinic networks with odd graph structures) can be observed on scaling up to large numbers of oscillators.

The phase oscillator approach can be used in applications where the dynamics of individual elements can be modelled as a limit cycle oscillator and that can be

\section{P. Ashwin ( $\otimes)$}

Mathematics Research Institute, College of Engineering, Mathematics and Physical Sciences University of Exeter, Exeter, EX4 4QF, UK

e-mail: P.Ashwin@exeter.ac.uk 
characterised by a scalar phase variable. This is particularly the case if there is weak coupling between the oscillators, or more specifically if the timescale associated with the coupling is much longer than the timescale associated with relaxation onto the limit cycle $[6,10,17]$. Moreover, phase models are typically useful even far from the weak coupling limit, especially concerning their predictions for generic bifurcations and attractors.

Clearly, the structure of coupling between oscillators is critical to determine what sort of dynamics is possible on a network of coupled oscillators, and there is a vast literature looking at the topology of coupling and the influence this has on the network dynamics; see for instance [1] and references therein. For the particular problem of synchronization this has been investigated by many authors, allowing for a variety of effects such as time-dependent coupling [7, 25] or delay in coupling $[19,32]$. For the main part of this paper we do not address such issues, but rather explore the question of generic but complicated dynamics that can appear even for very simple all-to-all (fully symmetric) coupling. Indeed, this emphasises that not only the network structure, but also the actual coupling function is vital in determining the emergent dynamics of the system.

In this paper we review some recent work on the detailed dynamics of globally coupled phase oscillators, concentrating on the appearance of robust heteroclinic network attractors. These attractors manifest themselves in the dynamics as a "persistent transient" of slow switching between a number of unstable states that is driven by noise and/or imperfections. Such dynamics were first discovered in [17] and further studies have been made in $[22,23]$, mostly working with large populations. In Sect. 2 we review the fundamental dynamical principles of coupled phase oscillator systems with permutation symmetry. In Sect. 3 we discuss a detailed study of bifurcations leading to sequential slow switching attractors and show that they are only possible for $N \geq 4$; see [4]. In Sect. 4 we present a new result arising from [8] showing that a particular combinatorial structure can appear in the dynamics for coupling as in [17], for open sets of system parameters and arbitrarily large $N$.

Consider $N$ identical phase oscillators that are coupled identically to each other

$$
\dot{\theta}_{i}=\omega+\frac{K}{N} \sum_{j=1}^{N} g\left(\theta_{i}-\theta_{j}\right)
$$

where the dot denotes differentiation with respect to time $t$ and $\theta_{i} \in \mathbb{T}=[0,2 \pi)$, $i=1 \ldots N$, i.e. the state space is the $N$-torus $\mathbb{T}^{N}$. We will use the vector notation $\theta=\operatorname{col}\left[\theta_{1}, \ldots, \theta_{N}\right]$. The function $g:[0,2 \pi) \rightarrow \mathbb{R}$ is a $2 \pi$-periodic coupling function that we will assume to be smooth. We include a coupling strength parameter $K$ for convenience but will set this to $K=1$ by rescaling time.

For a given coupling function $g$ and number of oscillators $N$, the dynamics of (1) can include a wide range of behaviours including in-phase (synchronous) oscillations, antiphase oscillations, and indeed arbitrarily complex partially synchronized cluster states that are stable [29]. However, system (1) can give much more complicated dynamics than just stable clustering. If the cluster states are of 
saddle type, robust heteroclinic connections may be found. This is because cluster states may have unstable manifolds contained within the stable manifold of another state; see, for example, [2, 17, 22]. This is not possible for the coupling function $g(\varphi)=-\sin (\varphi)$ of the Kuramoto model [24] or the coupling function $g(\varphi)=-\sin (\varphi+\alpha)$ of the Kuramoto-Sakaguchi model [3], but it is possible for slightly more complicated coupling functions.

Choosing the coupling function

$$
g(\varphi)=-\sin (\varphi+\alpha)+r \sin (2 \varphi)
$$

robust heteroclinic connections can be found that exist for open sets of parameter values on the $(\alpha, r)$ parameter plane [17]. Note that this coupling can be obtained from phase reduction of coupled Hodgkin-Huxley neurons with synaptic coupling [18]. More general couplings are considered for example in [12, 29]. We remark that system (1) can have highly nontrivial behaviour even for the Kuramoto coupling function if the frequencies are made non-identical; see [33]. In Sects. 3 and 4 we study the dynamics of certain parameter regimes for coupling (2), to illuminate the dynamics of system (1) and to better understand the graph structure of a possible heteroclinic network as $N$ increases.

\section{Dynamics and Bifurcations with $S_{N}$ Symmetry}

Here we review techniques from dynamical systems and bifurcation theory with symmetry $[16,15,26,27]$, noting that (1) is symmetric under all permutations of coordinates. In other words the dynamics is equivariant under the set $S_{N}$ of permutations acting by

$$
\sigma\left(\theta_{1}, \cdots, \theta_{N}\right)=\left(\theta_{\sigma(1)}, \cdots, \theta_{\sigma(N)}\right)
$$

In addition to this, the system (1) has a symmetry given by translation of all components by the same phase shift: for all $\rho \in[0,2 \pi)$ we have invariance under

$$
\rho\left(\theta_{1}, \cdots, \theta_{N}\right)=\left(\theta_{1}+\rho, \cdots, \theta_{N}+\rho\right)
$$

meaning that the system has symmetry $S_{N} \times S^{1}$. This has many dynamical consequences, the most obvious of which is that there will be an in-phase or fully synchronized solution

$$
\theta_{i}(t)=\Omega t+r
$$

for $i=1, \cdots, N$ where $\Omega=\omega+g(0)$ and $r$ is constant. This solution may or may not be an attractor depending on its linear stability.

Another consequence of symmetry is that there will be a number of invariant subspaces forced by the symmetry; the most general of these being the "rotating 
block" structures discussed in [6,9]. We will focus on a particular case here; the $M$ cluster states, for $1 \leq M \leq N$.

We define an $M$-cluster partition $\mathcal{A}=\left\{A_{1}, \ldots, A_{M}\right\}$ of $\{1, \ldots, N\}$ to be a partition such that

$$
\{1, \ldots, N\}=\bigcup_{p=1}^{M} A_{p},
$$

where the $A_{p}$ are pairwise disjoint sets $\left(A_{p} \cap A_{q}=\emptyset\right.$ if $\left.p \neq q\right)$. Summing up the cluster sizes $a_{p}=\left|A_{p}\right|$ gives

$$
\sum_{p=1}^{M} a_{p}=N .
$$

Denoting the phase of the $p$-th cluster by $\psi_{p}$, i.e. defining $\psi_{p}:=\theta_{i}=\theta_{j}=$ $\theta_{k}=\ldots$ such that $\{i, j, k, \ldots\} \subset A_{p}$ we obtain

$$
\dot{\psi}_{p}=\omega+\frac{1}{N} \sum_{q=1}^{M} a_{q} g\left(\psi_{p}-\psi_{q}\right),
$$

for $p=1, \ldots, M$. The dynamics in such a subspace can be very complex, but initially we investigate simple clustering behaviour

$$
\psi_{p}=\Omega t+\phi_{p},
$$

for $p=1, \ldots, M$, where $\Omega \in \mathbb{R}^{+}$and $\phi_{p} \in \mathbb{T}$. Since $\theta$ describes the phases of oscillators, (9) describes a periodic orbit in the state space of those oscillators.

Note that typical points in such a cluster state have symmetries obtained by all permutations within a cluster, i.e. an $M$-cluster state with partition $\mathcal{A}$ will have in terms of spatial symmetries

$$
S_{a_{1}} \times \cdots \times S_{a_{M}} .
$$

If there are any spatiotemporal symmetries these must be of the form of some cyclic permutation of clusters of equal size: they must be a semidirect product of permutations with the cycling

$$
\left(S_{a_{1}} \times \cdots \times S_{a_{\ell}}\right)^{k} \otimes \mathbb{Z}_{k},
$$

where the $M=k \ell$ clusters permuted in groups of $\ell$ and $k\left(a_{1}+\cdots+a_{\ell}\right)=N$ [6]. Note that the $S^{1}$ action is such that one can reduce the full dynamics of (1) to the dynamics of the phase differences and a translation on the $S^{1}$ orbit (group orbit). This means that, for example, the periodic orbits of (1) can be found by examining equilibria of the phase differences. 
Linearisations of systems near equilibria with symmetries have structure forced upon them by the presence of symmetry; they must be block-diagonal with respect to the isotypic decomposition of the tangent space with respect to the group that fixes the equilibrium (the isotropy subgroup of the equilibrium). As a consequence, generic bifurcations with any symmetry group will have a centre manifold on which the action of the isotropy subgroup is an irreducible representation (irrep) $[15,16]$. The permutation action of $S_{N}$ on $\mathbb{T}^{N}$ neatly splits into two irreps, one which is a 1-dimensional trivial action and one which is an irreducible $(N-1)$-dimensional action. (There are other nontrivial irreps for $S_{N}$ but they do not appear in (1).) Moreover, the nontrivial irrep corresponds precisely to the phase difference coordinates.

The generic $S_{N}$ symmetry breaking bifurcations for the nontrivial irrep have been studied and classified; see, for example, [15]. However, the topology of the torus $\mathbb{T}^{N}$ can and does easily associate local symmetry breaking bifurcations with global bifurcations, as we will see in Sect. 3; see also [3, 6]. Typically, $S_{N}$ symmetry breaking bifurcation from a stable fully symmetric state will result in a system with no nearby stable branches after bifurcation; this is associated in our system with global bifurcations that may lead to a stable cluster state or (only for $N \geq 4$ ) a robust heteroclinic attractor.

Note that periodic solutions of (1) with symmetry (11) can be interpreted in terms of cluster states in state space; for instance,

- $S_{N}$ corresponds to in-phase solutions $\theta_{k}=\Omega t+\gamma, k=1, \ldots, N$

- $\mathbb{Z}_{N}$ corresponds to anti-phase or "splay phase" solutions $\theta_{k}=\Omega t+\gamma+2 k \pi / N$, $k=1, \ldots, N$

- $\left(S_{N / 2}\right)^{2} \otimes \mathbb{Z}_{2}$ corresponds to antiphase solutions such as $\theta_{k}=\Omega t+\gamma, \theta_{k+N / 2}=$ $\Omega t+\gamma+\pi, k=1, \ldots, N / 2$

where $\Omega \in \mathrm{IR}+$ and $\gamma \in \mathbb{T}_{j}$ see (5) and (9).

Finally, we note that symmetry means that cluster states will appear in conjugate families; if a particular clustering is realised as a periodic orbit then so will all permutations of that clustering. This means that subspaces with conjugate isotropy subgroups will support identical dynamics.

\section{Bifurcations for Three and Four Globally Coupled Oscillators}

Returning to the specific system $(1,2)$, one can calculate the bifurcation behaviour in detail for small numbers of oscillators; this was done for $N=3$ and $N=4$ in [3] and here we briefly summarise some of the results, in particular with the aim of identifying where heteroclinic networks appear in parameter and state space.

Figure 1 shows the bifurcation diagram for $N=3$ in the $(r, \alpha)$ plane and Table 1 describes briefly each of the lines of codimension-one bifurcations (both reproduced from [3]). The bifurcation diagram is shown surrounded by phase portraits in the $\left(\theta_{1}-\theta_{2}, \theta_{1}-\theta_{3}\right) \in[0,2 \pi)^{2}$ plane. 

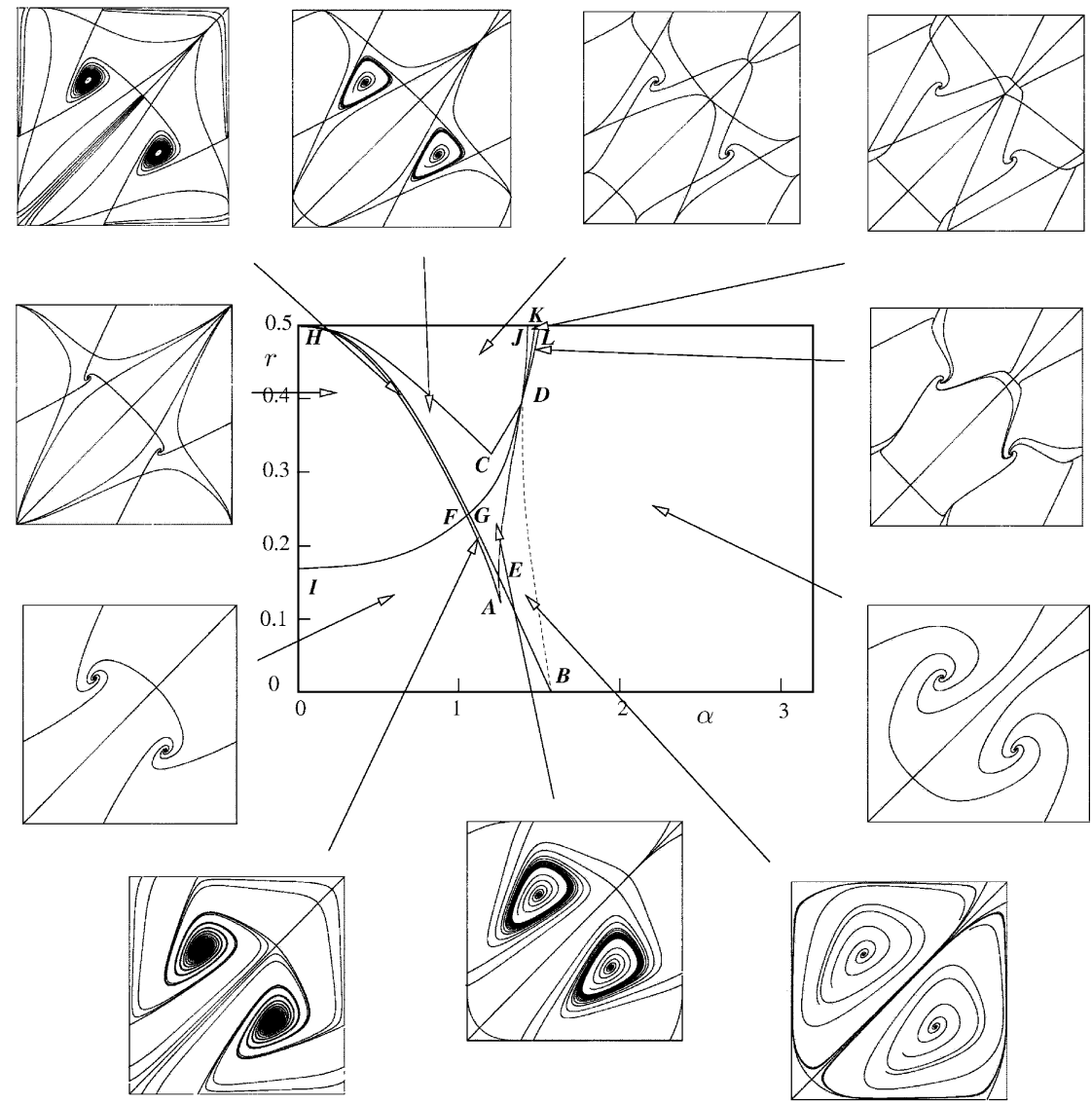

Fig. 1 Bifurcation curves in the $(r, \alpha)$ parameter plane for system (1) with coupling (2) in case of $N=3$ (reproduced from [3]). The phase portraits are shown for $\left(\theta_{1}-\theta_{2}, \theta_{1}-\theta_{3}\right) \in[0,2 \pi)^{2}$. The codimension-one bifurcations are listed in Table 1. Note that there are heteroclinic/homoclinic cycles only on the lines $\mathrm{BE}, \mathrm{ED}$ and $\mathrm{HCD}$ for $N=3$. There are codimension-two bifurcations: $A$ - cusp point; $E$ - interaction of transcritical homoclinic and saddle-node homoclinic; $D$ interaction of saddle-node heteroclinic and saddle connection heteroclinic; $H$ - degenerate Hopf bifurcation of antiphase

Moving on to the case for $N=4$ oscillators, there are 12 invariant regions corresponding to points on $\mathbb{T}^{4}$ that lift to the set

$$
\left\{\left(\theta_{1}, \theta_{2}, \theta_{3}, \theta_{4}\right): \theta_{\sigma(1)} \leq \theta_{\sigma(2)} \leq \theta_{\sigma(3)} \leq \theta_{\sigma(4)} \leq \theta_{\sigma(1)}+2 \pi\right\}
$$

for permutations $\sigma \in S_{4}$. Figure 2 illustrates one of these invariant tetrahedra plotted in the $\left(\theta_{1}-\theta_{2}, \theta_{1}-\theta_{3}, \theta_{1}-\theta_{4}\right) \in[0,2 \pi)^{3}$ space.

In Fig. 3 a bifurcation diagram is shown for the case $N=4$ in the $(r, \alpha)$ plane, while Table 2 lists the codimension-one bifurcations (both reproduced from [3]). 
Table 1 The letters label the curves of codimension-one bifurcations for $N=3$ illustrated in Fig. 1 (reproduced from [3])

\begin{tabular}{ll}
\hline$I D$ & Pitchfork bifurcation on invariant lines \\
$B E G H$ & Transcritical bifurcation at 0 \\
$H F A E D$ & Saddle-node bifurcation on invariant lines \\
$B E$ & Transcritical homoclinic bifurcation \\
$E D$ & Saddle-node homoclinic bifurcation \\
$B D$ & Saddle-node of limit cycles \\
$H C D$ & Saddle connection heteroclinic bifurcation \\
$D J$ & Pitchfork bifurcation \\
$D K$ & Saddle-node bifurcation \\
$D L$ & Saddle-node bifurcation \\
\hline
\end{tabular}

Observe that there is a similar set of bifurcations as in Fig. 3. In particular, for $r$ small and $-\pi / 2<\alpha<\pi / 2$ we have stable in-phase solutions while for $r$ small and $\pi / 2<\alpha<3 \pi / 2$ we have stable antiphase solutions. For both $N=3$ and $N=4$ the point $(r, \alpha)=(0, \pi / 2)$ acts as an "organizing centre" for a number of lines of bifurcations that coincide at this point. Note that the Kuramoto-Sakaguchi coupling function (Eq. (2) with $r=0$ ) is highly degenerate at the point $\alpha=\pi / 2$.

In the shaded region BEDTLV of Fig. 3, the dynamics consists of robust heteroclinic cycles between symmetrically related cluster states with $S_{2} \times S_{2}$ symmetry.

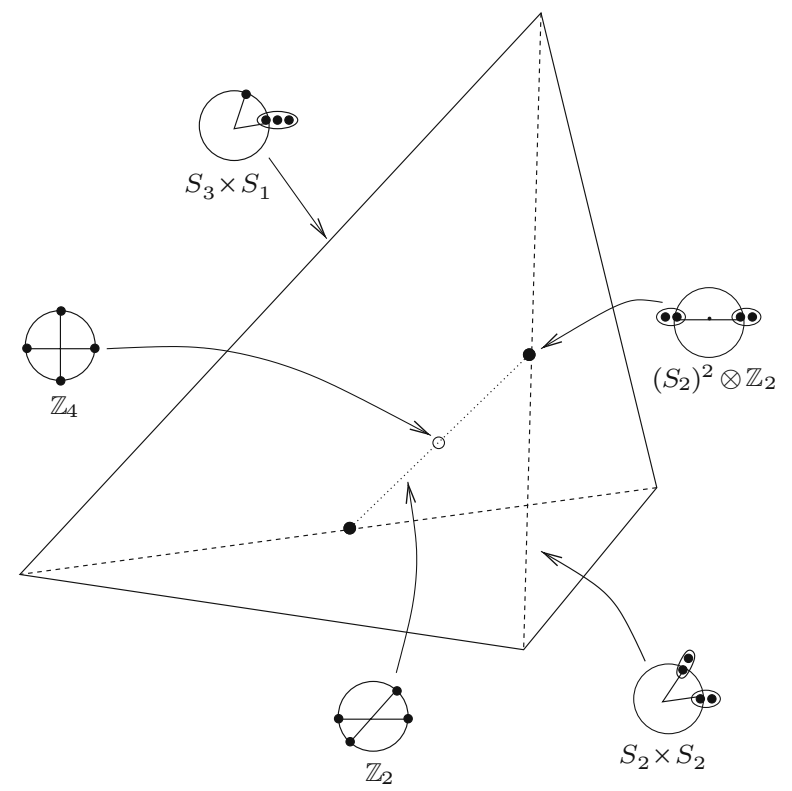

Fig. 2 Diagram showing one of the invariant regions (12) in state space for $N=4$ oscillators (reproduced from [3]). This shows the relationship between the subspaces with differing symmetries. The point at the centre is the antiphase solution with $\mathbb{Z}_{4}$ symmetry and the faces of the invariant tetrahedron is made of points with $S_{2}$ symmetry 


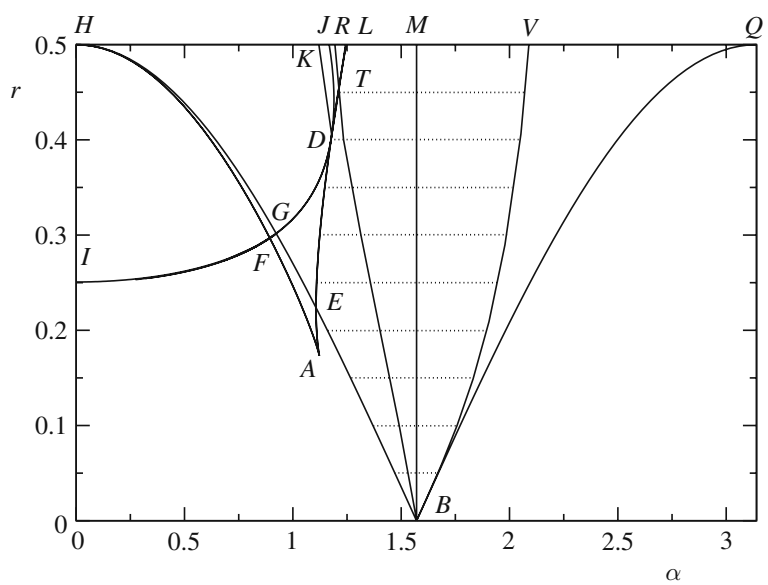

Fig. 3 Bifurcation diagram for $N=4$ oscillators in the $(\alpha, r)$ plane (adapted from [3]); see Table 2 for a description of the bifurcation curves. There are robust heteroclinic cycles between 2-cluster states in the shaded region outlined by BEDTLV. These cycles are attractors to the left of the line $B M$. There is a complicated sequence of bifurcations in the near vicinity of point $D$ that not is discussed here in detail

These cycles are attracting to the left of the curve $B M$. The $S_{2} \times S_{2}$ symmetry corresponds to 2-cluster states where each cluster contains two oscillators. For example, the partition

$$
\mathcal{A}=\{\{1,2\},\{3,4\}\},
$$

gives $\left[a_{1}, a_{2}\right]=[2,2]$ and the corresponding cluster phases can be defined as

$$
\begin{aligned}
& \psi_{1}:=\theta_{1}=\theta_{2}, \\
& \psi_{2}:=\theta_{3}=\theta_{4} .
\end{aligned}
$$

Table 2 A list of codimension-one bifurcations for $N=4$ that are illustrated in Fig. 3 (reproduced from [3])

\begin{tabular}{ll}
\hline$B E G H$ & Transcritical-pitchfork bifurcation at 0 \\
$B Q$ & Inverse pitchfork bifurcation of saddles at the point with $\left(S_{2}\right)^{2} \otimes \mathbb{Z}_{2}$ symmetry \\
$B V$ & Pitchfork/heteroclinic bifurcation of solutions with symmetry $S_{2} \times S_{2}$ (in transversal \\
& to $S_{2} \times S_{2}$ direction) \\
$B M$ & Hopf bifurcation of antiphase points $\mathbb{Z}_{4}$ and change of stability of robust heteroclinic \\
& $\quad$ cycles
\end{tabular}


Investigating the simple clustering behaviour (9) in these subspaces one may find the cluster states

$$
\left[\psi_{1}, \psi_{2}\right]=\Omega t+[a, b], \quad\left[\psi_{1}, \psi_{2}\right]=\Omega t+[b, a],
$$

where only the phase difference $a-b$ can be determined. By dropping $\Omega t$ we introduce the notation

$$
P_{1}=[a, a, b, b], \quad P_{2}=[b, b, a, a],
$$

for these cluster states.
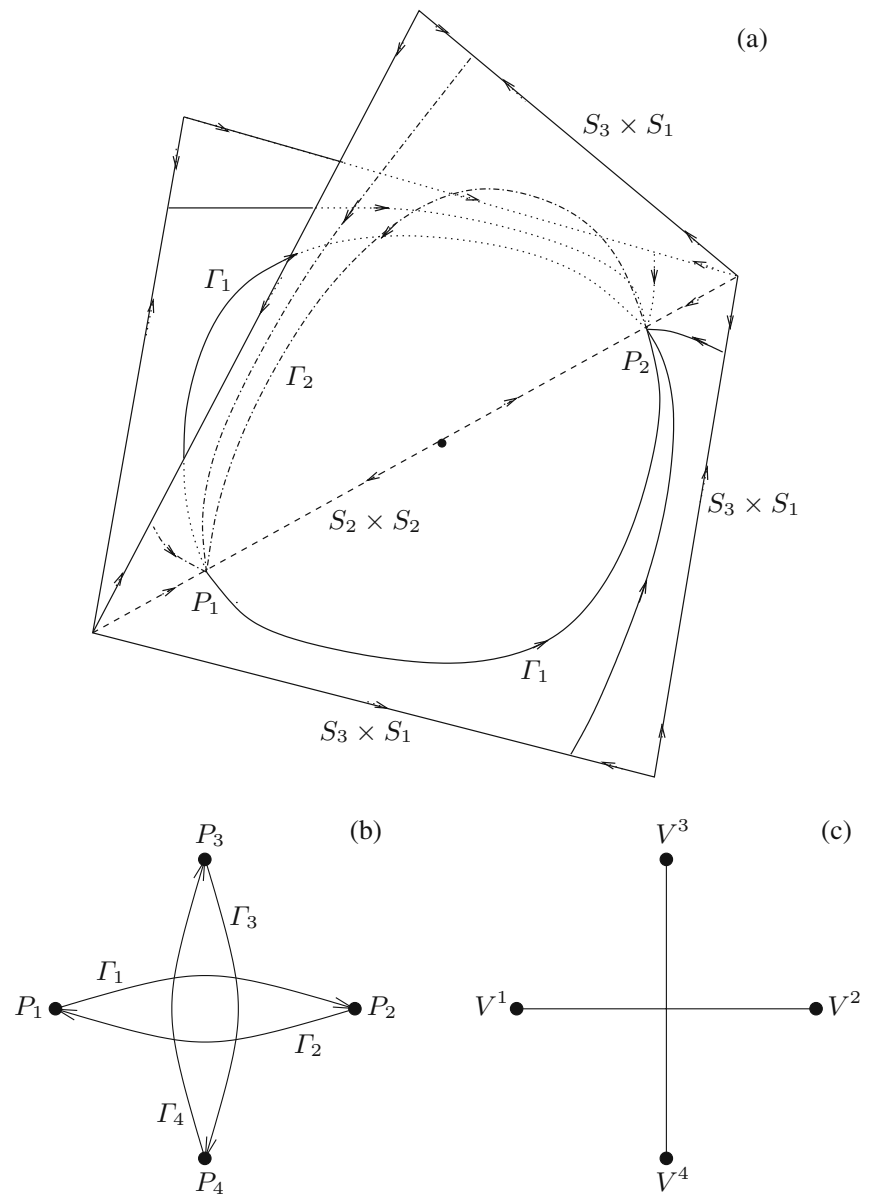

Fig. 4 Heteroclinic cycles for $N=4$ oscillators. Panel (a) shows the detailed dynamics involving the saddle cluster states $P_{1}, P_{2}(16)$ (adapted from [3]). The cycle between states $P_{1}, P_{2}$ (16) and the cycle between states $P_{3}, P_{4}(17)$ are shown schematically in panel (b). Panel (c) represents the network as an undirected graph where the vertices $V^{1}, \ldots, V^{4}$ correspond to cluster states $P_{1}, \ldots, P_{4}$ and edges are drawn between them when heteroclinic connections exist. The parameters are from the shaded area BEDTLV in Fig. 3 
One may find that the clusters with phase $b$ are unstable to perturbations that split the oscillators and there exist heteroclinic orbits $\Gamma_{1}$ and $\Gamma_{2}$ that connect $P_{1}$ and $P_{2}$. Figure 4a illustrates this dynamics in state space (adapted from [3]). One may also find a similar cycle between the 2-cluster states

$$
P_{3}=[a, b, a, b], \quad P_{4}=[b, a, b, a] .
$$

corresponding to the partition

$$
\tilde{\mathcal{A}}=\{\{1,3\},\{2,4\}\} \text {. }
$$

The heteroclinic cycles are sketched in Fig. $4 \mathrm{~b}$ and the corresponding graph structure of the network is shown in Fig. 4c. The vertex $V^{m}$ represents the cluster state $P_{m}$ and vertices are connected by edges when there are heteroclinic connections between the corresponding cluster states. Observe that there exist switching dynamics between cluster states but the network structure is trivial: the heteroclinic network splits into two disconnected components. For $N \geq 5$ the network becomes connected and much more complex as will be shown in the next section.

\section{Heteroclinic Networks for Odd Numbers of Oscillators}

Here we consider some of the scaling properties of the robust heteroclinic networks discussed in the previous section, for more larger numbers of oscillators. As noted in [8] for odd numbers of oscillators, $N=2 k+1(k \geq 2)$, one can obtain open regions of parameter space in which there are heteroclinic networks with the structure of an odd graph; in this paper we characterise this statement in detail and find the corresponding regions in parameter space numerically.

An odd graph of order $k$ is a family of partition-defined graphs defined as follows. Let the vertex $V^{m}$ represent a possible 2-cluster partition $\mathcal{A}^{m}=\left\{A_{1}^{m}, A_{2}^{m}\right\}$ of $N=$ $2 k+1$ when $\left[a_{1}^{m}, a_{2}^{m}\right]=[k, k+1]$. We call such partitions $[k, k+1]$ cluster states; they clearly have $S_{k} \times S_{k+1}$ symmetry. There are $m=1, \ldots, N ! /(k !(k+1) !)$ such vertices. We say there is an edge from a vertex $V^{m}$ to a vertex $V^{n}$ if

$$
A_{1}^{n} \subset A_{2}^{m}
$$

i.e. if the larger cluster of $\mathcal{A}^{m}$ contains the smaller cluster of $\mathcal{A}^{n}$. This graph is highly connected and each vertex has $k+1$ outgoing edges corresponding to which element in $A_{2}^{m}$ that is not in $A_{1}^{n}$. The high level of connectivity of these graphs has resulted in suggestions to use the structure for fault-tolerant networks; see, for example, [14]. Examples of odd graphs of orders $k=3$ and $k=4$ are shown in Fig. 5.

We demonstrate that for system (1) with coupling (2) and any odd $N=2 k+1$, $k=2,3, \ldots$ there is an open sets of parameters near $r=0$ and $\alpha=\pi / 2$ such that there is a heteroclinic network that contains the structure of an odd graph of 

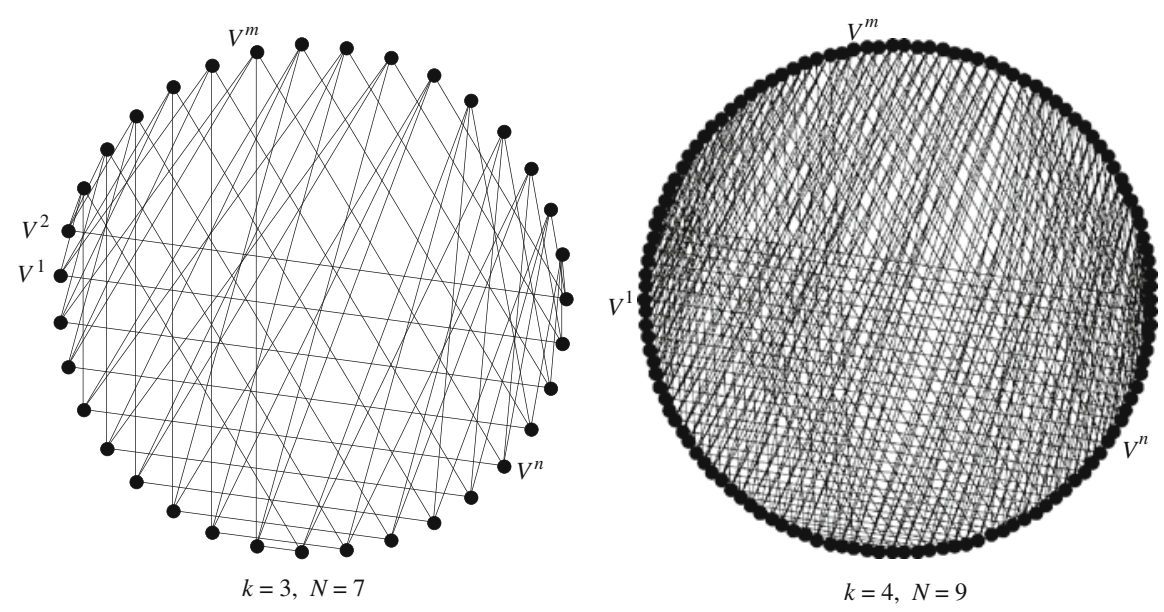

Fig. 5 Odd graphs of order $k=3$ and $k=4$; these structures are robustly present in heteroclinic networks of the coupled oscillator system (1) with coupling function (2) for $N=7$ and $N=9$ oscillators, respectively. The number of vertexes are 35 and 126, respectively, and they are presented in an analogous way to Fig. $4 \mathrm{c}$

order $k$. This network is robust to perturbations of the coupling function $g$ that are sufficiently small in the $C^{2}$ norm.

First, we show that two distinct families of $[k, k+1]$ cluster states may exist. We call these $a b$ cluster states and $c d$ cluster states, as will be clarified in Sect. 4.1; see also [2]. The $a b$ cluster states are unstable to perturbations of the smaller cluster while the $c d$ cluster states are unstable to perturbations of the larger cluster. Then we show that there may be heteroclinic connections from $a b$ cluster states to $c d$ cluster states and also from $c d$ cluster states to $a b$ cluster states. This means that applying perturbations to the appropriate (unstable) clusters one may navigate from a $a b$ cluster state to another $a b$ cluster state through a $c d$ cluster state. Such navigation between the $m$-th and $n$-th $a b$ cluster states is possible if and only if there is an edge between vertices $V^{m}$ and $V^{n}$ on the corresponding odd graph of order $k$; see Fig. 5. Notice that the odd graphs are not directed graphs: in fact if it is possible to navigate from the $m$-th $a b$ cluster state to the $n$-th one, then it is possible to navigate the other way, through a different $c d$ cluster state.

\subsection{Existence, Stability and Connections of $[k, k+1]$ Cluster States}

Consider the 2-cluster partition

$$
\mathcal{A}^{1}=\{\{1, \ldots, k\},\{k+1, \ldots, 2 k+1\}\}
$$


so that $\left[a_{1}, a_{2}\right]=[k, k+1]$ (that is indeed a $[k, k+1]$ cluster state) and define the cluster phases as

$$
\begin{aligned}
& \psi_{1}:=\theta_{1}=\ldots=\theta_{k} \\
& \psi_{2}:=\theta_{k+1}=\ldots=\theta_{2 k+1} .
\end{aligned}
$$

From (8), the equations for the time evolution of the cluster phases become

$$
\begin{aligned}
& \dot{\psi}_{1}=\omega+\frac{1}{N}\left(a_{1} g(0)+a_{2} g\left(\psi_{1}-\psi_{2}\right)\right), \\
& \dot{\psi}_{2}=\omega+\frac{1}{N}\left(a_{1} g\left(\psi_{2}-\psi_{1}\right)+a_{2} g(0)\right) .
\end{aligned}
$$

Considering the periodic orbit (9) and introducing the notation $\phi:=\phi_{1}-\phi_{2}$ formula we have

$$
0=a_{1}(g(0)-g(-\phi))+a_{2}(g(\phi)-g(0))
$$

to determine the phase difference $\phi$.

Linearizing (22) one may obtain the tangential stability which has a trivial eigenvalue $\lambda_{1}=0$ and a tangential eigenvalue

$$
\lambda_{2}=\frac{1}{N}\left(a_{1} g^{\prime}(-\phi)+a_{2} g^{\prime}(\phi)\right),
$$

corresponding to perturbations that do not split either of the clusters. The linearization of (1) gives the transverse eigenvalues

$$
\begin{aligned}
& \lambda_{3}=\frac{1}{N}\left(a_{1} g^{\prime}(0)+a_{2} g^{\prime}(\phi)\right), \\
& \lambda_{4}=\frac{1}{N}\left(a_{1} g^{\prime}(-\phi)+a_{2} g^{\prime}(0)\right),
\end{aligned}
$$

where $\lambda_{3}$ corresponds to splitting of the cluster of $k$ and has multiplicity $k-1$ while $\lambda_{4}$ corresponds to splitting of the cluster of $k+1$ and has multiplicity $k$.

Note that the dynamics in subspace (21) corresponding to partition $\mathcal{A}^{1}$ is effectively one-dimensional. Defining the variable $\psi:=\psi_{1}-\psi_{2}$ and subtracting the second equation from the first one in (22) results in

$$
\dot{\psi}=\frac{1}{N}\left(a_{1}(g(0)-g(-\psi))+a_{2}(g(\psi)-g(0))\right) .
$$

There is an equilibrium $\psi(t) \equiv \phi$ determined by (23) while the related eigenvalue is given by (24). Substituting (2) and $N=2 k+1$ into (26) and considering the large $k$ limit we have 


$$
\dot{\psi}=-\cos \alpha \sin \psi+r \sin (2 \psi)+\mathcal{O}\left(\frac{1}{2 k+1}\right) .
$$

Now consider the 2-cluster partition

$$
\mathcal{A}^{2}=\{\{k+2, \ldots, 2 k+1\},\{1, \ldots, k+1\}\}
$$

for which $\left[\tilde{a}_{1}, \tilde{a}_{2}\right]=[k, k+1]$ and the corresponding cluster phases can be defined as

$$
\begin{aligned}
& \tilde{\psi}_{1}:=\theta_{k+2}=\ldots=\theta_{2 k+1}, \\
& \tilde{\psi}_{2}:=\theta_{1}=\ldots=\theta_{k+1} .
\end{aligned}
$$

The evolution of these phases are still given by (22) so one may find the same equilibria with the same stability properties as for partition $\mathcal{A}^{1}(20)$.

According to (19), since $\{k+2, \ldots, 2 k+1\} \subset\{k+1, \ldots, 2 k+1\}$ then on the odd graph of order $k$ there is an edge connecting the vertices $V^{1}$ and $V^{2}$ (that represent the partitions $\mathcal{A}^{1}$ (20) and $\mathcal{A}^{2}$ (28), respectively). We show that there is a heteroclinic connection from the $a b$ cluster state with partition $\mathcal{A}^{1}$ to the $c d$ cluster state with partition $\mathcal{A}^{2}$ and that there is a connection from the $c d$ cluster state with partition $\mathcal{A}^{2}$ to the $a b$ cluster state with the same partition. Thus one may navigate from the $a b$ cluster state with partition $\mathcal{A}^{1}$ to the $a b$ cluster state with partition $\mathcal{A}^{2}$ through the $c d$ cluster state with partition $\mathcal{A}^{2}$.

Thus, we are interested in parameter regions where $\mathbf{A}, \mathbf{B}$ and $\mathbf{C}$ hold:

A There exist two different cluster states in the subspace (21) such that

$$
\left[\psi_{1}, \psi_{2}\right]=\Omega t+[a, b], \quad\left[\psi_{1}, \psi_{2}\right]=\Omega t+[c, d],
$$

that are both $[k, k+1]$ cluster states with partition $\mathcal{A}^{1}(20)$. They are called $a b$ and $c d$ cluster states and by dropping the $\Omega t$ we loosely say that in a $a b$ cluster state $k$ oscillators have phase $a$ and $k+1$ oscillators have phase $b$ while in a $c d$ cluster state $k$ oscillators have phase $c$ and $k+1$ oscillators have phase $d$. Indeed, only the phase differences

$$
\phi^{a b}=a-b, \quad \phi^{c d}=c-d,
$$

can be determined from (23) and by symmetry, such periodic orbits exist for all other $[k, k+1]$ cluster states with different partitions (e.g. with $\mathcal{A}^{2}(28)$ ).

B We require that the $a b$ cluster states are unstable to perturbations of the larger cluster of $b$-s and the $c d$ cluster states are unstable to perturbation of the smaller cluster of $c$-s while all other perturbations decay. This can be characterised in saying that we wish to find $\alpha, r$ such that for $a b$ cluster states we have $\lambda_{2}<0$, $\lambda_{3}<0, \lambda_{4}>0$ while for $c d$ cluster states we have $\lambda_{2}<0, \lambda_{3}>0, \lambda_{4}<0$; see (24) and (25). 
$\mathbf{C}$ We wish to find a subregion of $\mathbf{A}, \mathbf{B}$ in parameter space such that there is a heteroclinic connection from the $a b$ cluster state with partition $\mathcal{A}^{1}$ (20) to the $c d$ cluster state with partition $\mathcal{A}^{2}(28)$ and a heteroclinic connection from the $c d$ cluster state with partition $\mathcal{A}^{2}$ to the $a b$ cluster state with partition $\mathcal{A}^{2}$.

Steps A, B above are routine in terms of root finding and then evaluating the stabilities but step $\mathbf{C}$ is more complex. Note that (similarly to the $N=4$ case in Fig. 4a) there is an additional [ $k, k+1]$ cluster state in the subspace (21) but that is unstable against tangential perturbations $\left(\lambda_{2}>0\right)$ and hence does not appear in the heteroclinic network.

First let us search for the $a b \rightarrow c d$ heteroclinic connection by examining the partition

$$
\mathcal{B}^{1}=\{\{1, \ldots, k\},\{k+1\},\{k+2, \ldots, 2 k+1\}\}
$$

that corresponds to (by abuse of notation) $\left[b_{1}, b_{\mathrm{s}}, b_{2}\right]=[k, 1, k]$. Defining the cluster phases as

$$
\begin{aligned}
& \psi_{1}:=\theta_{1}=\ldots=\theta_{k}, \\
& \psi_{\mathrm{s}}:=\theta_{k+1}, \\
& \psi_{2}:=\theta_{k+2}=\ldots=\theta_{2 k+1},
\end{aligned}
$$

leads to

$$
\begin{aligned}
& \dot{\psi}_{1}=\omega+\frac{1}{N}\left(b_{1} g(0)+b_{\mathrm{s}} g\left(\psi_{1}-\psi_{\mathrm{s}}\right)+b_{2} g\left(\psi_{1}-\psi_{2}\right)\right), \\
& \dot{\psi}_{\mathrm{s}}=\omega+\frac{1}{N}\left(b_{1} g\left(\psi_{\mathrm{s}}-\psi_{1}\right)+b_{\mathrm{s}} g(0)+b_{2} g\left(\psi_{\mathrm{s}}-\psi_{2}\right)\right), \\
& \dot{\psi}_{2}=\omega+\frac{1}{N}\left(b_{1} g\left(\psi_{2}-\psi_{1}\right)+b_{\mathrm{s}} g\left(\psi_{2}-\psi_{\mathrm{s}}\right)+b_{2} g(0)\right),
\end{aligned}
$$

see (8). Notice that for $\psi_{\mathrm{s}}=\psi_{2}$ one obtains a $[k, k+1]$ cluster state with partition $\mathcal{A}^{1}$ (20) and (21), While for $\psi_{s}=\psi_{1} a[k, k+1]$ cluster state with partition $A^{2}$ (28) and (29) is obtained. One can numerically verify that there exists a heteroclinic connection between the $a b$ cluster state $\left[\psi_{1}, \psi_{\mathrm{s}}, \psi_{2}\right]=\Omega t+[a, b, b]$ and the $c d$ cluster state $\left[\psi_{1}, \psi_{\mathrm{s}}, \psi_{2}\right]=\Omega t+[d, d, c]$. Figure 6 shows such connections for $k=10(N=21)$ with parameters $\alpha=1.52$ and $r=0.1$. To eliminate $\Omega t$ the phase differences $\psi_{1}-\psi_{\mathrm{s}}$ and $\psi_{\mathrm{s}}-\psi_{2}$ are used.

Now let us search for the $c d \rightarrow a b$ connection by examining the partition

$$
\mathcal{B}^{2}=\{\{k+2, \ldots, 2 k\},\{2 k+1\},\{1, \ldots, k+1\}\},
$$

that corresponds to $\left[\tilde{b}_{1}, \tilde{b}_{\mathrm{s}}, \tilde{b}_{2}\right]=[k-1,1, k+1]$. Now defining the cluster phases as 


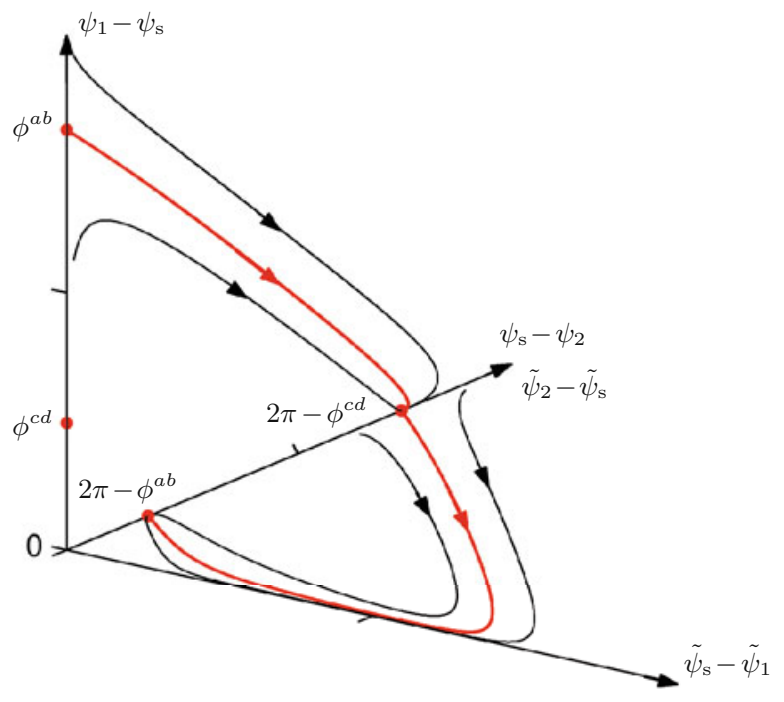

Fig. 6 Heteroclinic connections (thick curves) connecting $a b$ and $c d$ cluster states (dots) for $k=10$ $(N=21)$. The figure shows that one may navigate from the $a b$ cluster state with partition $\mathcal{A}^{1}(20)$ to the $a b$ cluster state with partition $\mathcal{A}^{2}$ (28) through the $c d$ cluster state with partition $\mathcal{A}^{2}$, that is, the sequence of connections $a b \rightarrow c d \rightarrow a b$ corresponds to an edge between the vertices $V^{1}$ and $V^{2}$ (representing $\mathcal{A}^{1}$ and $\mathcal{A}^{2}$ ) on the odd graph of order $k$, see Fig. 5. Additional trajectories (thin curves) demonstrate that the connections are attracting, that is, the heteroclinic connections are sinks within the relevant subspaces. The parameters are $\alpha=1.52$ and $r=0.1$ correspond to the cross in Fig. $7 d$

$$
\begin{aligned}
& \tilde{\psi}_{1}:=\theta_{k+2}=\ldots=\theta_{2 k}, \\
& \tilde{\psi}_{\mathrm{s}}:=\theta_{2 k+1}, \\
& \tilde{\psi}_{2}:=\theta_{1}=\ldots=\theta_{k+1} .
\end{aligned}
$$

leads to the same set of equations as (34) for $\tilde{\psi}_{p}$-s but with the current set of $\tilde{b}_{p}$-s. Note that for $\tilde{\psi}_{\mathrm{s}}=\tilde{\psi}_{1}$ a $[k, k+1]$ cluster state is obtained with partition $\mathcal{A}^{2}(28)$ and (29).

We can numerically verify that there exist heteroclinic connections between the $c d$ cluster state $\left[\tilde{\psi}_{1}, \tilde{\psi}_{\mathrm{s}}, \tilde{\psi}_{2}\right]=\Omega t+[c, c, d]$ and the $a b$ cluster state $\left[\tilde{\psi}_{1}, \tilde{\psi}_{\mathrm{s}}, \tilde{\psi}_{2}\right]=$ $\Omega t+[a, a, b]$ as shown in Fig. 6 for $k=10(N=21)$ with parameters $\alpha=1.52$, $r=0.1$. Now the phase differences $\tilde{\psi}_{\mathrm{s}}-\tilde{\psi}_{1}$ and $\tilde{\psi}_{2}-\tilde{\psi}_{\mathrm{s}}$ are used to represent the connection. Note that in Fig. 6 only the plane spanned by $\psi_{1}-\psi_{\mathrm{s}}$ and $\psi_{\mathrm{s}}-\psi_{2}$ and 

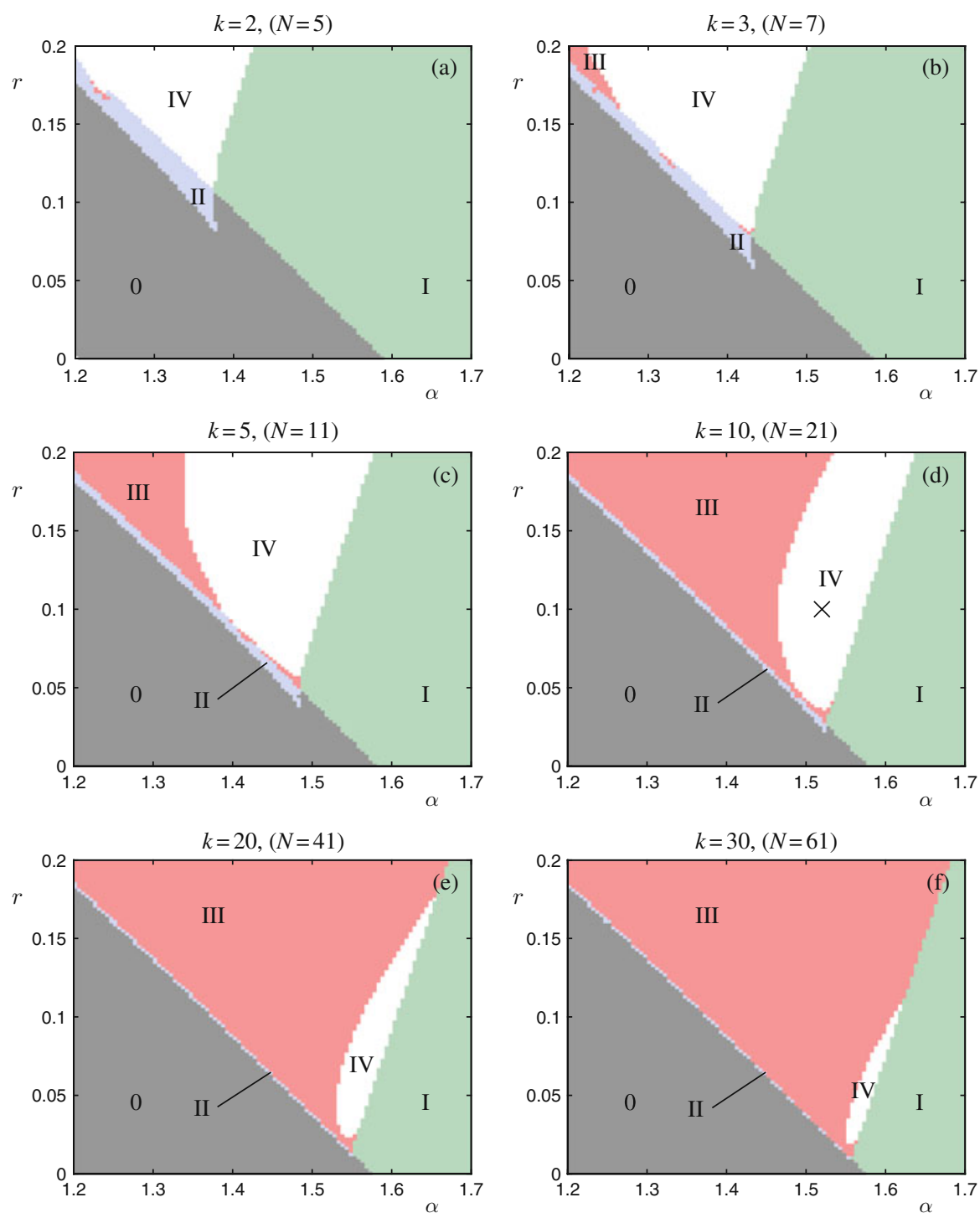

Fig. 7 In $(\alpha, r)$ parameter space the white region IV shows where the $N=2 k+1$ oscillator system $(1,2)$ has a heteroclinic network between $[k, k+1]$ cluster states with the structure of the odd graph of order $k$ for (a) $k=2$, (b) $k=3$, (c) $k=5$, (d) $k=10$, (e) $k=20$ and (f) $k=30$. The other regions indicated are as follows. Dark grey region 0 - neither cluster state exists; light green region I - only $a b$ cluster states exist; and light blue region II - only $c d$ cluster states exist; red region III - both type of cluster states exist but connections are missing. Note that the white region is open (confirming that the cycle is robust) but becomes smaller and moves towards $(\alpha, r)=(\pi / 2,0)$ on increasing $k$. The cross in panel (d) corresponds to the simulation in Fig. 6 
the plane spanned by $\tilde{\psi}_{\mathrm{s}}-\tilde{\psi}_{1}$ and $\tilde{\psi}_{2}-\tilde{\psi}_{\mathrm{s}}$ are used by the dynamics; the plane spanned by $\psi_{1}-\psi_{\mathrm{s}}$ and $\tilde{\psi}_{\mathrm{s}}-\tilde{\psi}_{1}$ is transverse to the dynamics.

For values of parameters $\alpha, r$ and $k$ where $\mathbf{A}, \mathbf{B}$ hold one may run finite time simulations of system (34) and determine whether the above described $a b \rightarrow c d$ and $c d \rightarrow a b$ connections exist. In this way the existence of a heteroclinic network equivalent in structure to an odd graph of order $k$ can be it verified numerically. This could in principle be done to a finite precision, provided that a bound on the errors is also calculated. In Fig. 7, a $100 \times 100$ mesh is used over the parameter regime $\alpha \in(1.2,1.7), r \in(0,0.2)$ and at each mesh-point it is checked whether the conditions $\mathbf{A}, \mathbf{B}$ and $\mathbf{C}$ are satisfied for $k$ ranging from $k=2(N=5)$ up to $k=30$ $(N=61)$.

Region IV (white) shows where A, $\mathbf{B}$ and $\mathbf{C}$ hold while region III (red) shows where only A, B hold but not $\mathbf{C}$. Regions I (light green) and II (light blue) are where A, B are only partially satisfied, i.e. only $a b$ or $c d$ cluster states exist, respectively. Finally, considering parameters from region 0 (dark grey) no condition hold, that is no cluster states can be found. Observe that the white region IV shrinks as $k$ increases.

Although this is not a rigorous proof that the odd graph structure is present for arbitrary (finite) $k$, it does suggest a means of verifying this. It may be possible to analytically prove the existence of an open non-empty region in the $(\alpha, r)$ parameter plane satisfying $\mathbf{A}, \mathbf{B}$ and $\mathbf{C}$ by using rigorous singular perturbation methods with a small parameter $\epsilon=1 / k$.

\section{Discussion}

We have shown that remarkably complicated bifurcations and structures may emerge in the dynamics of (1). In particular, robust heteroclinic networks can appear that lead to slow sequential switching between cluster states. We found that the number of oscillators needs to satisfy $N \geq 5$ to give rise to nontrivial robust switching dynamics between cluster states. The reason for this is that 5 is the minimum number where there is a nontrivial asymmetric partition, namely a [2,3] cluster state. Such a state has two clusters of differing size that can have different transverse stabilities, and this permits a nontrivial switching between different 2-cluster partitions. In general for $N=2 k+1$ oscillators, we determined parameter regimes where $[k, k+1]$ cluster states are connected to form a heteroclinic network with the structure of an odd graph of order $k$. We remark that for $N \geq 7$ the odd graph heteroclinic network is only a subnetwork of the full attracting heteroclinic network. The full network includes connections not just between cluster states of type $[k, k+1]$ but also between cluster states of type [ $k-2 m, k+2 m+1]$ for a variety of $m$. Up to now we have only scratched the surface of the combinatorial complexity that may exist in such networks.

We note that it is possible to find rather different heteroclinic structures for a variety of different coupling functions. For example, considering 


$$
g(\varphi)=-\sin (\varphi+\alpha)+r \sin (2 \varphi+\beta)
$$

and $N=2 k+1(N \geq 5)$, it is possible to find robust heteroclinic cycles between $[k, k, 1]$ cluster states for open sets of parameters [5, 28, 34]. We also remark that (1) does not have any sort of variational structure or global Lyapunov function for general choices of $g$ (although it may do for specific cases of interest [11]). Lack of variational structure is clearly a necessary condition for the existence of heteroclinic connections in the dynamics.

On preserving the coupling structure but breaking the permutation symmetry by making the oscillators non-identical, the dynamics can still be efficiently explained with reference to the heteroclinic network [28, 34]. Moreover, for strong nonlinear coupling, one can explain extreme sensitivity to detuning of the frequencies of oscillators. This is where arbitrarily small differences in the frequencies give rise to loss of frequency locking and this depends apparently on the presence of robust heteroclinic attractors that are topologically non-trivial on $\mathbb{T}^{N}$ [4]. On breaking the symmetry of coupling structure by removing connections, the dynamics can be much more rich; see, for example [13, 30] and references within. However, heteroclinic connections between unstable states may still exist even when the coupling is not all-to-all leading to sequential switching $[1,20]$.

The switching dynamics occurring in networks provides some promising models for a variety of phenomena, especially for neural applications where synchrony tends to be frustrated due to the competing requirements of efficiency (a neural code needs a minimum of energy to be maintained) and robustness (a neural code should be insensitive to removal or malfunction of individual cells). Particular applications that may be of relevance to the models presented here include finite-state computation [2] and spatio-temporal code generation [28, 34].

Acknowledgement We gratefully acknowledge Oleksandr Burylko, Mike Field, Yuri Maistrenko, Jeff Moehlis, Marc Timme, Stuart Townley and John Wordsworth for discussions related to this work. We also thank Robin Chapman for originally pointing us to the literature on odd graphs. This research was supported by EPSRC under grant EP/C510771/1 and by the Institute for Collaborative Biotechnologies under grant DAAD19-03-D004 from the US Army Research Office.

\section{References}

1. M. Aguiar, P. Ashwin, A. Dias, and M. Field. Dynamics of coupled cell networks: synchrony, heteroclinic cycles and inflation. Preprint (2010).

2. P. Ashwin and J. Borresen. Encoding via conjugate symmetries of slow oscillations for globally coupled oscillators. Phys. Rev. E, 70(2), 026203 (2004).

3. P. Ashwin, O. Burylko, and Y. Maistrenko. Bifurcation to heteroclinic cycles and sensitivity in three and four coupled phase oscillators. Phys. D, 237(4), 454-466 (2008).

4. P. Ashwin, O. Burylko, Y. Maistrenko, and O. Popovych. Extreme sensitivity to detuning for globally coupled phase oscillators. Phys. Rev. Lett., 96(5), 054102 (2006).

5. P. Ashwin, G. Orosz, J. Wordsworth, and S. Townley. Dynamics on networks of cluster states for globally coupled phase oscillators. SIAM J. Appl. Dynam. Syst., 6(4), 728-758 (2007). 
6. P. Ashwin and J. W. Swift. The dynamics of $n$ weakly coupled identical oscillators. J. Nonlinear Sci., 2(1), 69-108 (1992).

7. I. Belykh, V. Belykh, and M. Hasler. Generalized connection graph method for synchronization in asymmetrical networks. Phys. D, 224(1-2), 42-51 (2006).

8. J. Borresen. Dynamical encoding in systems of globally coupled oscillators. PhD thesis, University of Exeter (2006).

9. E. Brown, P. Holmes, and J. Moehlis. Globally coupled oscillator networks. In E. Kaplan, J.E. Marsden, and K.R. Sreenivasan, editors, Perspectives and Problems in Nonlinear Science: A Celebratory Volume in Honor of Larry Sirovich. Springer, New York, pp. 183-215 (2003).

10. E. Brown, J. Moehlis, and P. Holmes. On the phase reduction and response dynamics of neural oscillator populations. Neural Comput., 16(4), 673-715 (2004).

11. A. C. C. Coolen, R. Kühn, and P. Sollich. Theory of Neural Information Processing Systems. Oxford University Press, Oxford (2005).

12. H. Daido. Generic scaling at the onset of macroscopic mutual entrainment in limit-cycle oscillators with uniform all-to-all coupling. Phys. Rev. Lett., 73(4), 760-763 (1994).

13. M. Field. Combinatorial dynamics. Dynam. Syst., 19(3), 217-243 (2004).

14. A. Ghafoor and T. R. Bashkow. A study of odd graphs as fault-tolerant interconnection networks. IEEE Trans. Comput., 40(2), 225-232 (1991).

15. M. Golubitsky and I. Stewart. The Symmetry Perspective: From Equilibrium to Chaos in Phase Space and Physical Space, Progress in Mathematics. Birkäuser, Berlin, vol. 200 (2002).

16. M. Golubitsky, I. Stewart, and D. G. Schaeffer. Singularities and Groups in Bifurcation Theory: Volume II, Applied Mathematical Sciences. Springer, New York, vol. 69 (1988).

17. D. Hansel, G. Mato, and C. Meunier. Clustering and slow switching in globally coupled phase oscillators. Phys. Rev. E, 48(5), 3470-3477 (1993a).

18. D. Hansel, G. Mato, and C. Meunier. Phase dynamics for weakly coupled Hodgkin-Huxley neurons. Europhys. Lett., 23(5), 367-372 (1993b).

19. E. M. Izhikevich. Phase models with explicit time delays. Phys.l Rev. E, 58(1), 905-908 (1998).

20. Ö. Karabacak and P. Ashwin. Heteroclinic ratchets in networks of coupled oscillators. J. Nonlinear sci., 20(1), 105-129 (2010).

21. I. Z. Kiss, C. G. Rusin, H. Kori, and J. L. Hudson. Engineering complex dynamical structures: sequential patterns and desynchronization. Science, 316(5833), 1886-1889 (2007).

22. H. Kori. Slow switching in a population of delayed pulse-coupled oscillators. Phys. Rev. E, 68(2), 021919 (2003).

23. H. Kori and Y. Kuramoto. Slow switching in globally coupled oscillators: robustness and occurrence through delayed coupling. Phys. Rev. E, 63(4), 046214 (2001).

24. Y. Kuramoto. Self-entrainment of a population of coupled non-linear oscillators. In H. Araki, editor, International Symposium on Mathematical Problems in Theoretical Physics, Lecture Notes in Physics. Springer, New York, vol. 69, pp. 420-422 (1975).

25. W. L. Lu, F. M. Atay, and J. Jost. Chaos synchronization in networks of coupled maps with time-varying topologies. Eur. Phys. J. B, 63(3), 399-406 (2008).

26. J. Moehlis and E. Knobloch. Equivariant bifurcation theory. Scholarpedia, 2(9), 2511 (2007a).

27. J. Moehlis and E. Knobloch. Equivariant dynamical systems. Scholarpedia, 2(10), 2510 (2007b).

28. G. Orosz, P. Ashwin, and S. Townley. Learning of spatio-temporal codes in a coupled oscillator system. IEEE Trans. Neural Netw., 20(7), 1135-1147 (2009).

29. G. Orosz, J. Moehlis, and P. Ashwin. Designing the dynamics of globally coupled oscillators. Prog. Theor. phys.,122(3), 611-630 (2009).

30. I. Stewart and M. Parker. Periodic dynamics of coupled cell networks II: cyclic symmetry. Dynam. Syst., 23(1), 17-41 (2008).

31. S. H. Strogatz. From Kuramoto to Crawford: exploring the onset of synchronization in populations of coupled oscillators. Phys. D, 143(1-4), 1-20 (2000).

32. M. Timme, F. Wolf, and T. Geisel. Prevalence of unstable attractors in networks of pulse-coupled oscillators. Phys. Rev. Lett., 89(15), 154105 (2002). 
33. L. S. Tsimring, N. F. Rulkov, M. L. Larsen, and M. Gabbay. Repulsive synchronization in an array of phase oscillators. Phys. Rev. Lett., 95(11), 014101 (2005).

34. J. Wordsworth and P. Ashwin. Spatio-temporal coding of inputs for a system of globally coupled phase oscillators. Phys. Rev. E, 78(6), 066203 (2008). 